\title{
Políticas internas e influencias externas en el debate sobre derechos humanos en América Latina
}

El debate sobre la situación de los derechos humanos en América Latina, particularmente en lo que respecta a los países del Cono Sur, ha s.do intenso en los últimos años, concentrándose principalmente en la naturaleza de las políticas adoptadas en el plano interno de cada país y la discusión sobre su legitimidad e ilegitimidad, sus alcances y sus efectos. Este aspecto del debate ha dado lugar a una extensa literatura, tanto de carácter político como de carácter propiamente humanitario ${ }^{1}$.

Pero hay también otra dimensión de esta cuestión, que consiste en la relación existente entre esas políticas internas y las influencias externas que se han manifestado sobre la región. Este ángulo del problema ha merecido, comparativamente, un menor grado de atención, aun cuando probablemente ha sido determinante de muchos de los enfoques prevalecientes en el ámbito nacional, tanto en un sentido negativo como positivo ${ }^{2}$. Este artículo se limitará a analizar algunas de las complejas interrelaciones entre esas influencias externas y las políticas internas, en las variadas manifestaciones que ellas se expresan.

\section{DEREGHOS hUMANOS Y PROBLEMAS DE TRANSGULTURIZACIÓN}

Un distinguido autor latinoamericano ha observado que los derechos humanos y su protección constituyen una creación de carácter

"Capitulo escrito para el libro Fuman Rights in Foreign Policy', editado por R. J. Vincent, que será publicado por el Royal Institute of International Affairs de Londres.

"Véase en general Claudio Orrego Vicuña: La dificil senda del desarrollo politico en América Latina. cisec. Santiago, 1983. Particularmente la bibliografía alli citada.

${ }^{2} \mathrm{E} 1$ Instituto de Estudios Internacionales de la Universidad de Chile organizó en 1978 un seminario sobre el problema de los derechos humanos en las relaciones internacionales, que llevó a cabo un extenso análisis del tema, con particular referencia a América Latina. Véase Derechos Humanos y Relaciones Internacionales. Instituto de Estudios Internacionales de la Universidad de Chile, Santiago, 1979. En lo sucesivo citado como Derechos Humanos y Relaciones. 
cultural, que está asociada a determinados períodos de la evolución histórica ${ }^{3}$, con particular referencia a la civilización occidental ${ }^{4}$. Si bien hoy día puede afirmarse que los derechos humanos representan un valor universalmente reconocido, no debe dejar de observarse que se trata ésta de una realidad más bien nominal, pues en muchas partes su grado de respeto y cumplimiento dista mucho de sersatisfactorio5. Ello es probablemente una consecuencia directa de los diferentes grados de cultura prevalecientes en las diversas regiones del mundo, a lo que están también asociadas las variadas ideologíaís en cuanto a una forma de expresión cultural.

En la medida en que las formas más perfectas de la cultura contemporánea van alcanzando un mayor grado de internacionalización, sus valores van adquiriendo paralclamente una más extensa aplicación. Difícil es, por cierto, idcntificar cuáles son esas expresiones culturales más perfectas, pues en este ámbito son varias las concepciones que rivalizan entre sí, las que a su vez tienen diferentes creencias en cuanto al papel del individuo y sus derechos. Sea como fuere, puede suponerse que la civilización cristiana occidental es una de estas culturas avanzadas, pues ha identificado claramente al individuo como uno de sus valores centrales, sin que tampoco quepa desconocer las dificultades serias que ha encontrado en garantizar una protección efectiva de sus derechos.

El problema más difícil es determinar en qué medida estos últimos valores han sido recibidos efectivamente en América Latina. Es bien sabido que esta región es el producto cultural de una compleja mezcla de civilizaciones y valores, en que se combinan los elementos propios del cristianismo occidental con los de valores y tradiciones indígenas y otros muchos componentes foráneos. Más allá de las apariencias, cabe preguntarse si los valores culturales de América Latina son los mismos que los cle Occidente, o difieren en algunos aspectos de importancia ${ }^{6}$.

En la imposibilidad de dar una respuesta cierta, puede a lo menos afirmarse que si bien prevalece en la sociedad latinoamericana la raíz cultural occidental, ella no guarda una identidad total y ha evolucionado de acuerdo a sus propias realidades ${ }^{7}$. Lo anterior sig-

"Jorge Millas: "Derechos humanos y diferencias culturales en el mundo". En Derechos Fumanos y R'elaciones. p. 40.

"Jorge Tván Hübner: "Derechos Humanos y Cultura Occidental". En Derechos Humanos y Relaciones. p. 30.

"Antonio Bascuñán y Enrique Barros: "Hacia una transculturización de los derechos humanos". En Derechos FHumanos y Relaciones. p. 21.

"Para una discusión más general del problema, Francisco Orrego Vicuña: "América Latina: ¿Clase Media de las. Naciones?". Revista Estudios Intermacionales, publicada por el Instituto de Estudios Internacionales de la Universidad de Chile. No 40. Octubre-Diciembre de 1977.

"Francisco Orrego Vicuna: "Europe and South America: toward a complementary International. Role?". Western Europe: The Alliance in transition. Chicago Council on Foreign Relations. 198:1. 
nifica que siendo posible y necesario exigir el respeto de los derechos humanos fundamentales como valor superior de esa cultura, no puede ignorarse el contexto propio en que ella está inserta en América Latina, que es diferente de aquel que puede caracterizar a las naciones europeas o a los Estados Unidos, entre otras. De allí, que muchas veces procedan distinciones, que no siempre se han hecho, en la manera como debe enfocarse el problema, al menos mientras el proćeso señalado de la transculturización adquiere una dimensión más estable y profunda. Nada de lo anterior, ni aún en la medida en que existan diferencias, justifica violaciones o excesos en el campo de los derechos humanos, pues en muchos momentos América Latina ha sabido demostrar que puede ser tan riespetuosa de esos derechos como cualquier otra nación, pero sí requiere que se preste más atención a las causas que puedan haber llevado al actual deterioro y a las maneras de superarlas en forma permanente, lo que es una tarea esencialmente cultural en un amplio sentido.

\section{LAS DOCTRINAS DE LA SEGURTDAD NACIONAL Y SU ORIGEN OCGIDENTAL}

El problema de la transculturización que se comenta es todavía más complejo, pueș normalmente se supone que aquella cultura más perfecta realizará aportes positivos en cuanto al tema que nos preocupa del tratamiento de los derechos humanos. Sin embargo, ello no siempre ha sido así. En el caso de América Latina, está bien documentada la influencia que han tenido en el desarrollo de algunas visiones extremas de las doctrinas de la seguridad nacional las concepciones prevalecientes en determinados sectores de la sociedad francesa, norteamericana o, más remotamente, alemana ${ }^{8}$.

Los enfoques aplicados por Francia en el curso de la guerra de independencia de Argelia, por ejemplo, de los cuales no podría afirmarse que fueron un modelo de respeto de los derechos humanos, han tenido una fuerte influencia en América Latina en cuanto a su concepción totalizadora de lo bueno y de lo malo, de lo amigo y de lo enemigo, así como en cuanto a los métodos de eliminación total que fueron utilizados. Otro tanto ha ocurrido con las teorías relativas a la contrainsurgencia desarrolladas por los Estados Unidos, o con algunas concepciones maniqueas que fueron el producto de la guerra fría, en todas las cuales los ejércitos latinoamericanos fueron adiestrados sistemáticamente durante varias décadas. Un fenómeno similar es el que había ocurrido anteriormente con las escuelas geopolíticas alemanas.

De esta manera, el proceso de transculturización venía acompa-

"Genaro Arriagada: "Seguridad Nacional y Política". En Seguridad Nacional y Bien Común. CISEc, Santiago, 1976. p. 9. 
ñado de sus propias contradicciones, pues mientras juristas, hombres de iglesia o ciudadanos comunes aspiraban a que America Latina fuere el reflejo de valores e ideales superiores, otros segmentos de sus propias sociedades proyectaban concepciones antagónicas o valores opuestos. Nada de ello excluye, por cierto, la responsabilidad que cabe asignar a los extremos que se alcanzaron en América Latina, pues en definitiva hubo receptividad a esas ideas negativas, pero tampoco debe prescindirse de la responsabilidad que también cabe a esas otras sociedades en la génesis y desarrollo del problema.

Es incluso interesante observar que, luego de un período en que esas doctrinas se caracterizaron en América Latina por su exacerbación, que corresponde precisamente a la etapa más difícil en materia de derechos humanos, se pasó a una fase en que se realizan planteamientos moderados, más acordes con las tradiciones. latinoamericanas ${ }^{9}$. Quizás ello marca el punto en que se inicia una nueva separación entre el pensamiento latinoamericano y aquellas influencias externas, fenómeno que ha sido de frecuente ocurrencia en América Latina en muchos otros campos ${ }^{10}$.

\section{EXPERIENGIAS INTERNACIONALES NEGATIVAS Y FORMAS DE DEPENDENCTA HISTÓRICAS}

El problema que se analiza, debe situarse también en el contexto de una experiencia internacional más general que América Latina ha tenido a lo largo de su evolución histórica a partir de la Independencia. Esta experiencia no ha sido particularmente positiva, pues ha tenido como rasgo dominante el de una dependencia acentuada del sistema internacional y de sus potencias dominantes en diferentes períodos históricos, lo cual se ha traducido en un cierto sentido de desconfianza respecto de lo que puedan ser las intenciones reales de determinadas políticas auspiciadas por potencias $u$ otros países ajenos a la región ${ }^{11}$.

En una perspectiva histórica, esta situación afecta en primer lugar al caso de los paises europeos, que durante buena parte del siglo XIx ejercieron un papel protagónico dentro de un sistema internacional. El importante número de conflictos económicos, políti$\cos$ y militares que ocurrió entre potencias europeas y países de América Latina, es quizás sintomático de una relación que no podría exactamente definirse como de tipo cooperativo. Aun cuan-

"Horacio Toro I.: "Los derechos del individuo y del Estado: hacia un esquema de armonización". En Derechos Humanos y Relaciones, p. 58.

${ }^{10}$ Francisco Orrego Vicuña: "Democracia, pluralismo y sistema internacional: incidencia en la cooperación regional y latinoamericana". En Heraldo Muñoz V.: Politica exterior latinoamericana. A ser publicado por Westview Press.

"xara un análisis de la evolución histórica véase el artículo citado en la nota anterior, con particular referencia a los siglos $\mathrm{xIx} y \mathrm{xx}$. 
do es piobable que todo ello haya sido olvidado en Europa, no lo ha sido enteramente en América Latina.

Respecto de los Estados Unidos, estas dificultades han sido todavía más agudas, como consecuencia del papel hegemónico que este país ha ejercido en las relaciones continentales descle fines del siglo pasado. Lo que frecuentemente se ha tratado de definir como una relación especial y una comunidad de intereses, en el hecho ha sido una fórmula más bien retórica para encubrir discrepancias que con frecuencia han sido profundas y prolongadas en el tiempo ${ }^{12}$.

Si bíen la política exterior de los Estados Unidos ha alternado entre los ciclos célebres de aislacionismo e internacionalismo, para los efectos de la relación con América Latina ellos no se han traducido en cambios fundamentales, aun cuando puedan haber variado las modalidades a través de las cuales se procuran alcanzar los objetivos de esa política. De ahí que básicamente se haya percibido esta relación, por parte de los países latinoamericanos, como una expresión del fenomeno del imperialismo.

También debe tenerse presente que, con cierta frecuencia, la política exterior norteamericana ha patrocinado causas que forman parte de la tradicional moralidad de esa sociedad, adquiriendo así un sentido espiritual que en ocasiones se ha acercado a una visión mesiánica de la política internacional13. Con todo lo legítimo que ello es, este enfoque también se ha prestado a confusiones, temiéndose que tras las motivaciones humanitarias pudieran esconderse intenciones políticas de otra naturaleza. Lamentablemente, también la experiencia histórica avala estos temores.

\section{DERECHOS hUMANOS Y POLÍ́tICAS INTERNAGIONALES IMPOSITIVAS}

Los aspectos que se han examinado sirven de trasfondo para apreciar los diferentes efectos que han producido en América Latina los varios modelos de influencia internacional que se han intentado en. el plano de los derechos humanos en los últimos años. El primero de estos modelos fue el que llevó a cabo la Administración Carter. Si bien el problema de los derechos humanos ya se venía planteando con anterioridad, en etapas correspondientes a otros pexiódos solía no reaccionarse o sólo hacerse de manera marginal.

En este sentido, debe tenerse presente que, aparte de las muchas

\footnotetext{
15Véase el documento preparado por el Instituto de Estudios Internacionales: de la Universidad de Chile para el diálogo interamericano organizado por el Wilson Center en Washington, los días 15 y 16 de octubre de 1982: "El elusivo entendimiento entre América Latina y los Estados Unidos". En Esludios Internacionales. No 60. Octubre-Diciembre de 1982. p. 519.

${ }^{23}$ Walter Sánchez: "Imperialismo e idealismo en la polftica exterior norteamericana: el debate sobre los derechos humanos". En Derechos FIumanos y Relaciones. p. 85 .
} 
situaciones históricas de violación de derechos humanos que se han dado en América Latina, en la época contemporánea ha habido serias dificultades bajo dictaduras populistas como la de Perón, Pérez Jiménez o Rojas Pinillas, bajo dictaduras marxistas o bajo regimenes militares de derecha. De esta manera, el fenómeno venía manifestándose desde la década de 1950 con cierta regularidad, para alcanzar su major intensidad en la de 1970. Ello en sí mismo parece estar sugiriendo una deficiencia cultural de fondo.

La característica predominante del modelo de la Administración Carter fue la de fundamentarse en un enfoque impositivo, consistente principalmente en la aplicación de sanciones económicas y políticas. Tres fueron los principales problemas que este enfoque encontró en América Latina:

i) A pesar de que el Gobierno norteamericano procuró darle una interpretación positiva, en el sentido de que no se trataba de una medida de represalia sino que de una destinada a privar de los beneficios de una nueva prosperidad a los países afectados, en el ámbito latinoamericano ello sólo podía entenderse en su expresión más negativa. La experiencia histórica que se ha indicado lo hacia inevitable. Para peor, ese gobierno procedió a aplicar sanciones a los países latinoamericanos por muchas otras causas, ya se tratara de la política nuclear brasileña o de la política pesquera del Ecuador, entre otros ejemplos. Todo ello contribuyó a crear una relación de antagonismo general, que revivió las antiguas imágenes del imperialismo en la región.

ii) Una segunda dificultad fundamental surgió del hecho de que esa Administración introdujo en la discusión del tema un nuevo factor altamente conflictivo: la dimensión estratégica. La política sobre derechos humanos sería menos exigente cuando el país en cuestión representara un interés estratégico para los Estados Unidos en el campo político, económico o militar. De esta manera, el tema básico perdía enteramente su alcance humanitario para transformarse en una herramienta de promoción política de los intereses de una potencia internacional. Esta situación condujo a que esa política sobre derechos humanos perdiera en buena medida su credibilidad, incluso en los propios Estados Unidos.

iii) Como consecuencia de los dos problemas anteriores, en Anérica Latina se tendió a ver esta política como un problema de enfrentamiento político entre intereses nacionales contrapuestos y no como una cuestión que derivara de la necesaria salvaguardia de vitales valores morales. Por otra parte, la aplicación de esa política tampoco se distinguió claramente entre dos aspectos básicos, lo que se prestó a una mayor confusión todavía en cuanto a los alcances que ella tendría. El primer aspecto consistía en la protección de la integridad fisica de los individuos, en tanto que el segundo se 
refería a derechos más generales de orden económico o político ${ }^{14}$. Mięntras en lo primero se podía ser terminantemente exigente para asegurar su respeto, lo segundo admitía un mayor grado de discusión. Al no hacerse esta distinción, se pensó en muchos sectores gubernamentales que toda la intención de la referida política iba dirigida a un hostigamiento de los regímenes militares y no a una genuina salvaguardia de los derechos humanos básicos.

La discusión sobre los efectos que esta política ha tenido en América Latina ha sido intensa. Dos han sido los principales puntos de vista expresados sobre este particular. Para el primero de ellos, se trató de un esfuerzo exitoso que Iogró que en esta región se corrigieran algunos aspectos especialmente serios en el tratamiento de los derechos humanos ${ }^{15}$. Para el segundo, en cambio, se habrían generado efectos contraproducentes, como consecuencia de lo cual se habrían producido endurecimientos en la política seguida por algunos de los regímenes latinoamericanos.

Si bien resulta difícil apreciar la realidad de una situación tancontemporánea y reciente como es esta, el problema de los efectos de este modelo debe quizás enfocarse desde un doble punto de vista. El primero consiste en el de los alcances internacionales que podría tener una política sobre derechos humanos que fuese contradictoria con los postulados que patrocinaba en ese momento el Gobierno de los Estados Unidos. En ese contexto, no parece caber duda de que la amenaza de sanciones y la aprobación de decisiones condenatorias hizo sentir su peso en la región, Io que llevó a muchos gobiernos a adoptar una política más moderada sobre derechos humanos o a iniciar diversas formas de reordenamiento institucionaI16.

Pero, por otra parte, es necesario tener presente la evolución interna de los respectivos esquemas políticos. En la medida en que éstos parecían asentarse en el plano nacional, la situación general de los derechos humanos tendía proporcionalmente a mejorar, al igual que se hacía necesario proceder a las formas escogidas de ordenamiento institucional. Si esto es así, caben entonces dos preguntas. La primera es si acaso no se habría llegado de todos modos al mismo resultado como consecuencia de esa evolución interna, sin que pudieran atribuirse estos efectos a la presión internacional. Qui-

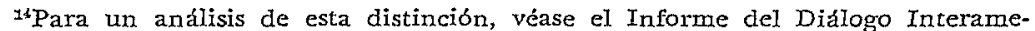
ricano organizado por el Wilson Center, publicado en el siguiente documento: "The Americas at a crossroads. Report of the Inter-American Dialogue, April 1983. Washington, The Wilson Center, 1983. p. 63.

${ }^{16}$ Claudio Orrego Vicuña: "Permanencia o transitoriedad en la estrategia de la Administración Carter: evaluación de dos años y perspectivas". En Derechos Humanos y Relaciones. p. 134.

${ }^{10}$ Instituto de Estudios Internacionales de la Universidad de Chile: Ensayos sobré la transición en América Latina. A ser publicado por Editorial de la Úniversidad de Belgrano, Buenos Aires.
} 
zás el hecho de que esta última coincidió con esa evolución, haga difícil atribuir el efecto a una u otra razón.

La segunda pregunta que emana de esta situación es más importante. ¿Obedeció esa mayor moderación a un convencimiento genuino o fue solamente el producto de una conveniencia circunstancial? En el primer caso, puede hablarse de un efecto positivo, ya sea su origen la evolución interna o la presión internacional. En el segundo, en cambio, su carácter circunstancial determinaría que se podría abandonar la nueva actitud tan pronto cambiasen las condiciones internas o externas. Dado el hecho de que los modelos impositivos no buscan el convencimiento o el consenso sino solamente Ios resultados, pudo temerse de que esta segunda alternativa fuese la más probable. En tal caso, se estaría frente a efectos negativos de la presión internacional, al menos en un largo plazo. Sin embargo, estas preguntas no encontraron una respuesta clara cn la práctica, pues las políticas de la Administración Carter fueron de corta duración y se produjo un cambio de modelo en este plano, aspecto que será examinado más adelante.

Cabe agxegar todavía un problema adicional, derivado de otros aspectos de la presión internacional, que fue particularmente evidente en la política seguida por Francia y algunos otros países. Se trata de la dimensión económica del enfoque aplicado. Puede apreciarse que existe, en este sentido, una relación inversamente proporcional entre la importancia asignada al tema de los derechos humanos y el nivel de la actividad comercial y de inversiones en el país en cuestión. Mientras algunas economías latinoamericanas registraron el "boom" de mediados de la década pasada ${ }^{17}$, el nivel de presión disminuyó ostensiblemente. Antes y después de ese período de prosperidad el interés por los derechos humanos fue sustantivamente mayor. Esta otra situación tampoco contribuyó a prestigiar esas políticas internacionales ni a fortalecer el convencimiento' de los regímenes latinoamericanos sobre los méritos del tema.

\section{DERECHOS HUMANOS Y POLÍTTGAS INTERNAGIONALES DISU'ASTYVAS}

Las dificultades internacionales que encontró el modelo anterior, que llevaron a los Estados Unidos a una situación de antagonismo generalizado en América Latina y en otras regiones del mundo, determinaron el surgimiento de un modelo alternativo. La política desarrollada bajo este otro modelo, fundamentándose en los mismos valores morales relativos a los derechos humanos, adopta una técnica diferente, que procura lograr un efecto disuasivo respecto de los

TTara un análisis de la influencia de la expansión económica en la política exterior de Chile, Heraldo Muñoz: "Las relaciones exteriores del Gobierno Militar chileno". En Chile 1973-1982. Revista Mexicana de Sociología, Facultad Latinoamericana de Ciencias Sociales, 1983. p. 229. 
excesos que se pudieran cometer en este campo. Para ello se utilizan las gestiones discretas y se evita el enfrentamiento público. De esta manera, el modelo confía conseguir tanto su objetivo humanitario como cautelar el interés político de los Estados Unidos sobre la base de evitar roces con países actual o potencialmente cercanos a ese interés.

La aplicación de este modelo por parte de la Administración Reagan no ha estado tampoco exenta de dificultades en América Latina, pudiendo mencionarse otros tres problemas principales:

i) El primero de estos problemas es que al desaparecer los elementos de presión pública respecto de la protección de los derechos humanos, algunos sectores parecieron interpretar que ello significaba una despreocupación por el tema de parte del Gobierno norteamericano. Sobre esta base pudo pensarse que nuevamente se padía actuar sin temor de represalias o sanciones internacionales y en la práctica se observó en algunos países un cierto deterioro en el plano de los derechos humanos. Esta situación suscitó también una nueva polémica en el propio plano político interno de los Estados Unidos.

Si bien, en realidad, la política de los Estados Unidos no había variado en cuanto al fondo, pues se fundamentaba en los mismos principios humanitarios en que descansa la sociedad norteamericana, el equívoco que se había producido hizo necesario reiterar su adhesión a los postulados de los derechos humanos. A comienzos de 1982 pudo apreciarse que se había restablecido el énfasis en el tema, después de un año de política silenciosa.

ii) La segunda dificultad estuvo nuevamente relacionada con el vínculo entre derechos humanos y aspectos estratégicos. Dada la importante prioridad que se asignó a estos últimos, tanto en lo que respecta a la política general frente a la Unión Soviética como al caso centroamericano en particular, algún gobierno latinoamericano pudo pensar en que su contribución estratégica significaría que se haría caso omiso de su situación en materia de derechos humanos $^{18}$. En cierto modo, se seguía la misma lógica estratégica que había aplicado la Administración Carter.

Este nuevo equívoco tuvo dos lamentables consecuencias. La primera consistió en el deterioro ya mencionado del cuadro sobre derechos humanos. La segunda consecuencia fue que ese régimen, creyendo que había alcanzado una alianza tácita con los Estados Unidos, desencadenó el conflicto del Atlántico Sur y precipitó una serịa crisis internacional, regional y nacional.

iii) EI tercer orden de dificultades es que ésta, como quizás cualquier otra política sobre derechos humanos, encontró límites dra-

${ }^{18} F r a n c i s c o$ Orrego Vicunia: "La crisis del Atlántico Sur y su influencia en el Sistema Regional". Estudios Internacionales. No 60. Octubre-Diciembre de 1982. p. 473 . 
máticos en cuanto a su eficacia en situaciones de guerra civil o violencia generalizada, como la que ha afectado a Nicaragua, El Salvador y Guatemala. Se creaba así un nuevo tipo de fenómeno que de alguna manera escapaba al ámbito de aplicación de esa política. Una consecuencia inevitable es que la política en su conjunto puclo haber resultado dañada por este problema.

También es interesante observar que la discusión sobre los efectos de esta política se ha planteado en los mismos términos que aquella relativa al modelo impositivo, pero con la diferencia de que se han invertido los argumentos. Para los sectores más bien conservadores, se ha tratado de un esfuerzo exitoso que ha permitido lo. grar sus objetivos de manera discreta y gradual. Para aquellos otros situados más a la izquierda, ha sido este un modelo fracasado que ha producido resultados contraproducentes.

Independientemente de los argumentos de carácter partidista, puede observarse que en América Latina se ha registrado recientemente una positiva evolución en este campo, tanto en lo que se refiere a la protección de la integridad física del individuo como al aspecto más general del proceso de redemocratización. El hecho de que todavía haya excepciones no altera la realidad de una tendencia general, como lo atestiguan los casos de Bolivia y Brasil y potencialmente los de Argentina y Uruaguay ${ }^{19}$.

El problema consiste, nuevamente, en determinar si acaso ello es el fruto de la misma evolución interna que se venía observando o bien se trata de un efecto atribuible a la existencia de una política internacional más efectiva. Si bien esta última puede ciertamente ayudar a este proceso, en el panorama actual no parecieran caber dudas de que se trata del resultado de una dinámica interna, ya sea de tipo cooperativo como ha ocurrido en Brasil, o de tipo conflictivo como ha sido el caso de Argentina. Sea como fuere, los límites del modelo incernacional se han hecho evidentes. Al mismo tiempo, tampoco cabe duda de que en este caso el proceso responde a un genuino convencimiento desarrollado en el ámbito de cada sociedad.

\section{DEREGHOS hUMANOS $Y$ POLÍtTCAS INTERNACIONALES SELEGTMVAS}

Mientras los modelos anteriores se desarrollaban principalmente en el marco de las relaciones bilaterales entre los gobiernos respectivos, paralelamente se estructuraban algunas políticas en el contexto de los mecanismos internacionales para la protección de los derechos del hombre, particularmente en Naciones Unidas ${ }^{20}$ y la Or-

"Para una reciente discusión del caso chileno, Hugo Fruhling, Garlos Portales y Augusto Varas: Estado y Fuerzas Armadas. Facultad Latinoamericana de Ciencias Sociales. Santiago, 1982. Véase también la obra citada en Nota 16 supra. ${ }^{30}$ Para un análisis general del sistema de Naciones Unidas y sus principales 
ganización de los Estados Americanos ${ }^{21}$. Sin perjuicio de ello, también han actuado en este plano diversos otros organismos internacionales de carácter público o privado.

Determinadas expresiones de estas otras políticas pueden agruparse en un modelo caracterizado por su selectividad. En este otro modelo, nuevamente se pudo apreciar la interferencia de los criterios políticos -ya sea respondiendo a líneas de política gubernamental o partidista- con los objetivos centrales de tipo humanitario que son propios de esos mecanismos internacionales de protección. Este fenómeno ha sido muy agudo en el caso de las Naciones Unidas y también ha podido apreciarse, con menos intensidad, en cl actuar de Ia Comisión Interamericana de Derechos Humanos.

Diversas dificultades aparecen asociadas con este enfoque. La primera de ellas es que en muchas oportunidades la selectividad obedeció simplemente a preferencias ideológicas, que llevó a un nuevo tipo de maniqueísmo, en que Ias violaciones de derechos humanos cometidas por regímenes de una determinada orientación resultaban condenables, en tanto que las cometidas por las de la orientación antagónica resultaban ignoradas o tratadas con singular deferencia. Ello condujo a que el actuar de estos mecanismos se percibiera como una herramienta de hostigamiento politico y no como una expresión de una genuina protección de los derechos fundamentales del hombre.

La segunda dificultad central fue que, desde el momento en que se entró en un juego político de esa naturaleza, se comenzó a evidenciar la estructuración de variadas alianzas y entendimientos fundamentados en la conveniencia de sus actores, independientemente de su respetabilidad en el plano de los derechos humanos. Quizás el caso más notorio fue el del entendimiento argentino-soviético, mediante el cual se garantizaba el no hostigamiento del primero en el ámbito de determinados mecanismos multilaterales a cambio de ventajas que obtenía el segundo en otras situaciones, incluidas las comerciales. De esta manera, la selectividad ideológica se vio todavía agravada con la discriminación en función de estrategias coyunturales.

Otra consecuencia de un enfoque en que tendía a predominar el interés político por sobre el fin humanitario, fue que nuevamente se borró la distinción entre las varias categorías de derechos humanos $y$, en particular, entre la protección de su integridad física y otros derechos inalienables o los más genéricos de tipo político y social. Ciertamente es un ideal que todos esos derechos se puedan

dificultades jurfdicas, Louis B. Sohn: "The improvent of the United Nations machinery on Human Rights". En Derechos Humanos y Relaciones. p. 171.

"Edmundo Vargas C.: "El perfeccionamiento de los mecanismos interamericanos a la luz de su experiencia". En Derechos Humanos y Relaciones. p. 222. 
salvaguardar conjuntamente, pero también es claro que en la práctica hay prioridades y valores que tienen más importancia que otros. La confusión que se introdujo en este plano, llevó a que se criticara por igual un acto abominable de tortura o desaparecimiento de un individuo y el modelo de política económica liberal que se aplicaba en el Cono Sur.

Las exageraciones en que incurrió este modelo fueron enteramente contraproducentes para la eficacia de sus políticas y en buena medida influyeron en disminuir el prestigio de esos respectivos mecanismos internacionales. Lo que debió ser un criterio humanitario estrictamente aplicado a todo quien transgrediera esos derechos, cualquiera fuese su ideología, peso o influencia, se transformó en una pugna política circunstancial aprovechada para fines partidistas.

Debe sí agregarse, con toda claridad, que no siempre los organismos internacionales actuaron dentro del enfoque indicado, pues ha habido algunos que se atuvieron estrictamente a sus fines humanitarios. Entre éstos cabe señalar, por ejemplo, el caso del Comité Intergubernamental para las Migraciones, la Cruz Roja Internacional, Amnesty International o varias entidades dependientes de las iglesias cristianas. Aun cuando muchas veces se adoptaron posiciones duras o informes severos, que ciertamente no fueron del agrado de los respectivos gobiernos, la predominancia del interés humanitario o el alto sentido de imparcialidad profesional de sus dirigentes, les imprimió un sello de autoridad que está al margen de todo debate político. En esa misma medida, su influencia se ve sin duda acrecentada.

\section{Derechos humanos $x$ LÍMTtes de LA POLÍtTaA EXTERIor}

Hay todavía otra dimensión internacional del problema de los derechos humanos que es menester analizar, la cual se relaciona, a diferencia de los modelos anteriores, con la política exterior de los propios países que se han visto afectados por una política adversa en este plano u otros aspectos asociados ${ }^{22}$. Es un hecho bien comprobado que esas políticas exteriores han encontrado crecientes límites en cuanto a su eficacia y capacidad de alcanzar respuestas apropiadas para las necesidades del país en cuestión. Esta situación puede apreciarse en tres escalas diferentes, pero que por cierto están asociadas.

En primer término, se ha observado una cierta tendencia al deterioro en las relaciones bilaterales y la situación vecinal entre países que se han visto afectados por los referidos problemas. Ello parece ser una consecuencia Iógica de la predominancia de elemen-

"2raldo Muñoz. loc. cit. Nota 17 supra. 
tos vinculados al extremo nacionalismo es alguno de estos paísès; que así como en el plano interno pueden utilizar de la violencia en contra de los individuos, también en el plano externo manifiestan similares inclinaciones. La política belicista que han patrocinado algunos sectores argentinos en contra de Chile, por ejemplo, es una expresión de este problema.

Una segunda escala de este fenómeno se puede apreciar en el plano regional, respecto del cual también se observa el mismo proceso de deterioro. Las dificultades y crisis en que se encuentra la cooperación latinoamericana y sus respectivas organizaciones regionales, es una consecuencia de la existencia de actitudes antagónicas que muchas veces se relacionan con esas visiones nacionalistas. La desvinculación clel ámbito latinoamericano que han experimentado varios de los países que cuentan con regímenes militares es otra expresión de este problema. De la misma manera, las amenazas a la paz y a la estabilidad que emanan de las tensas relaciones vecinales suelen contagiar al conjunto o buena parte de una región, que se transforma en un medio altamente conflictivo.

El tercer ámbito donde el mismo tipo de problema vuelve a ocurrir es en el plano más general de las relaciones internacionales. Ya sea porque se debilitan las relaciones bilaterales con algunas potencias de importancia mundial, o porque la región en su conjunto pierde fuerza en sus modalidades de inserción internacional, el hecho es que también se suele pasar de formas de relacion cooperativa a otras de carácter conflictivo. En algunas ocasiones, este carácter conflictivo llega a provocar serias tensiones para la paz internácional, como lo revela claramente el caso del Atlántico Sur y el de 'Centroamérica.

Las limitaciones que derivan de todo lo anterior para las respectivas políticas exteriores son sumamente severas. Aun cuando muchas veces ello no se percibe por los gobiernos afectados, o se atribuye a causas de otro tipo, en el mediano y largo plazo se produce un efecto sumamente dañino para los intereses nacionales que se encuentran comprometidos. En la medida en que se tenga conciencia de ello, quizás puedan. extraerse las conclusiones apropiadas para: el perfeccionamiento de las políticas de derechos humanos y para evitar los excesos negativos del extremo nacionalismo.

\section{LEGCTONES DE UNA EXPERIENCIA Y NECESTDADES DE REFORMULACIÓN}

Los diferentes aspectos que se han analizado, sugieren diversas conclusiones de importancia a la luz de la experiencia reciente de América Latina en este campo, que a la vez pueden servir de fundamento para reformular aquellas políticas que se hayan demostrado equivocadas. 
i) La primera conclusión es la evidente necesidad de definir criterios objetivos para la aplicación de las políticas internacionales de protección de los derechos humanos, sobre cuya base exista la certeza de cuáles son las condiciones y modalidades que pondrán en marcha los respectivos mecanismos. Esto incluye la conveniencia de asignar prioridades a los que constituyen derechos humanos fundamentales y distinguirlos de otros tipos de derechos más generales. No se trata de que se disminuya la importancia de estos últimos, pero al distinguirse entre diversas categorías se evita que cualquier confusión pueda terminar afectando a esos otros derechos fundamentales, como se ha visto en el caso latinoamericano.

ii) Dentro del mismo espiritu del punto anterior, es esencial que toda la política se inspire exclusivamente en los propósitos humanitarios que son propios del tema, excluyéndose en la medida de lo posible las interferencias de carácter político partidista y coyuntural. Estos últimos criterios pueden ser muy legítimos como parte de una pugna política, pero al utilizarse en conjunto con esas otras finalidades humanitarias se tiende inevitablemente a confundirlas con las luchas de tipo partidista, en circunstancias que debieran estar por encima de toda otra consideración. En la experiencia latinoamericana, esta utilización política del tema humanitario ha sido una de las situaciones que ha perjudicado más la efectividad de las variadas políticas internacionales que se han intentado.

iii) Un tercer aspecto, que también es parte del criterio de objetividad, es la necesidad de evitar aplicaciones selectivas y discriminatorias, sobre todo cuando ello se hace por razones de conveniencia política, como ha sido el caso de algunas organizaciones internacionales. Ello no solamente es arbitrario, sino que también es sumamente contraproducente para los fines que se persiguen, pues en definitiva se tiende a descartar de plano la intervención de los mecanismos que han utilizado estos enfoques. Especial importancia debe asignarse en este campo a la aplicación universal de los criterios elaborados, independientemente de toda consideración de tipo ideológico.

iv) La experiencia latinoamericana también parece sugerir la conveniencia de que no se ponga tanto énfasis en los aspectos represivos y sancionadores de estas políticas internacionales, pues también tienden a producir una reacción adversa, muchas veces asociada a Ios antiguos recuerdos del imperialismo o el colonialismo. Es más importante otorgar prioridad a los aspectos positivos que podrían desarrollarse, especialmente aquellos que contribuyan a incremen. tar el convencimiento genuino acerca de la necesidad de proteger los derechos humanos. Como se señaló en un comienzo, el problema en cuestión tiene una raíz muy profunda de tipo cultural, que la historia latinoamericana ha dejado en evidencia durante muchos decenios. Estas dificultades culturales sólo pueden superarse sobre 
la base de un enfoque positivo. Consensualismo y no imposición es también una regla válida en este campo.

v) Finalmente, también es importante tener presente que las políticas internacionales que se han comentado, sobre todo los modelos más discutidos, no deben traducirse en la creación de nuevas formas de dependencia de América Latina respecto de los centros de poder mundial, como en alguna medida ha ocurrido. Estas políticas sólo pueden tener éxito si acaso se diseñan de manera compatible con las necesidades y crecientes tendencias de Ia autonomía latinoamericana frente a la sociedad internacional.

Lamentablemente, muchas veces este aspecto no se ha tomado en consideración de manera suficiente, llegando, en algunas visiones extremas, a sugerirse que tales políticas podrian justificar formas de intervención por parte de potencias internacionales para salvaguardar sus objetivos humanitarios. Este punto de vista involucra un total desconocimiento de la tradición latinoamericana y sus actuales requerimientos de autonomía.

Enfocadas correctamente, varias políticas internacionales serían concebibles y podrian contribuir muy positivamente al perfeccionamiento de la protección de los derechos humanos, como de hecho ha ocurrido en el caso de algunas organizaciones internacionales en que han predominado el sentido profesional y la imparcialidad en su quehacer. 